\title{
IL-7 and IL-15 combined with strong TCR stimulation decrease Treg suppressive activity in healthy donors and patients with rheumatoid arthritis
}

\author{
Daniil Shevyrev ${ }^{1}$, Valeriy Tereshchenko ${ }^{1}$, Alexey Sizikov ${ }^{1}$, and Vladimir Kozlov ${ }^{1}$ \\ ${ }^{1}$ FSBSI Research Institute of Fundamental and Clinical Immunology
}

December 10, 2020

\begin{abstract}
Homeostatic proliferation (HP) is a physiological process to reconstitute the T-cell pool after lymphopenia with IL-7 and IL-15 being the key cytokines regulating the process. However, there is no evidence whether these cytokines influence the function of regulatory $\mathrm{T}$ cells (Tregs). Since lymphopenia often accompanies autoimmune diseases, we decided to study the proliferation rate and function of Tregs stimulated by IL-7 and IL-15 in patients with rheumatoid arthritis (RA) compared to healthy donors (HD). The study used peripheral blood from 14 RA patients and 18 HD. Proliferation of purified CD $3^{+} \mathrm{CD} 4{ }^{+} \mathrm{CD} 25^{+} \mathrm{CD} 127^{\mathrm{lo}}$ cells was assessed by flow cytometry using CFSE. Tregs were stimulated by anti-CD3, IL-7, IL-15, IL-7, or IL-15 combined with anti-CD3, and by IL- $2+$ anti-CD3, and their functional activity was evaluated in each case by CD4 ${ }^{+}$and CD $8^{+}$cells proliferation inhibition. The suppressive activity of peripheral Tregs did not differ between RA and HD; however, it significantly decreased when IL-7 or IL-15 were applied together with strong TCR stimulation with anti-CD3 antibodies. Herewith Treg proliferation caused by IL-7 and IL-15 was lower in RA than in HD. The revealed decrease in Treg suppressive activity can lead to the proliferation of potentially self-reactive T-cell clones, which can receive relatively strong TCR signals. This may be another explanation of why lymphopenia is associated with the development of autoimmune diseases. The revealed decrease of Treg proliferation under IL-7 and IL-15 may lead to a delay in Treg pool reconstitution in patients with rheumatoid arthritis.
\end{abstract}

\section{Hosted file}

DV Shevyrev IL7 and IL15 reduce Treg function.pdf available at https://authorea.com/users/ 382146/articles/498114-il-7-and-il-15-combined-with-strong-tcr-stimulation-decreasetreg-suppressive-activity-in-healthy-donors-and-patients-with-rheumatoid-arthritis 


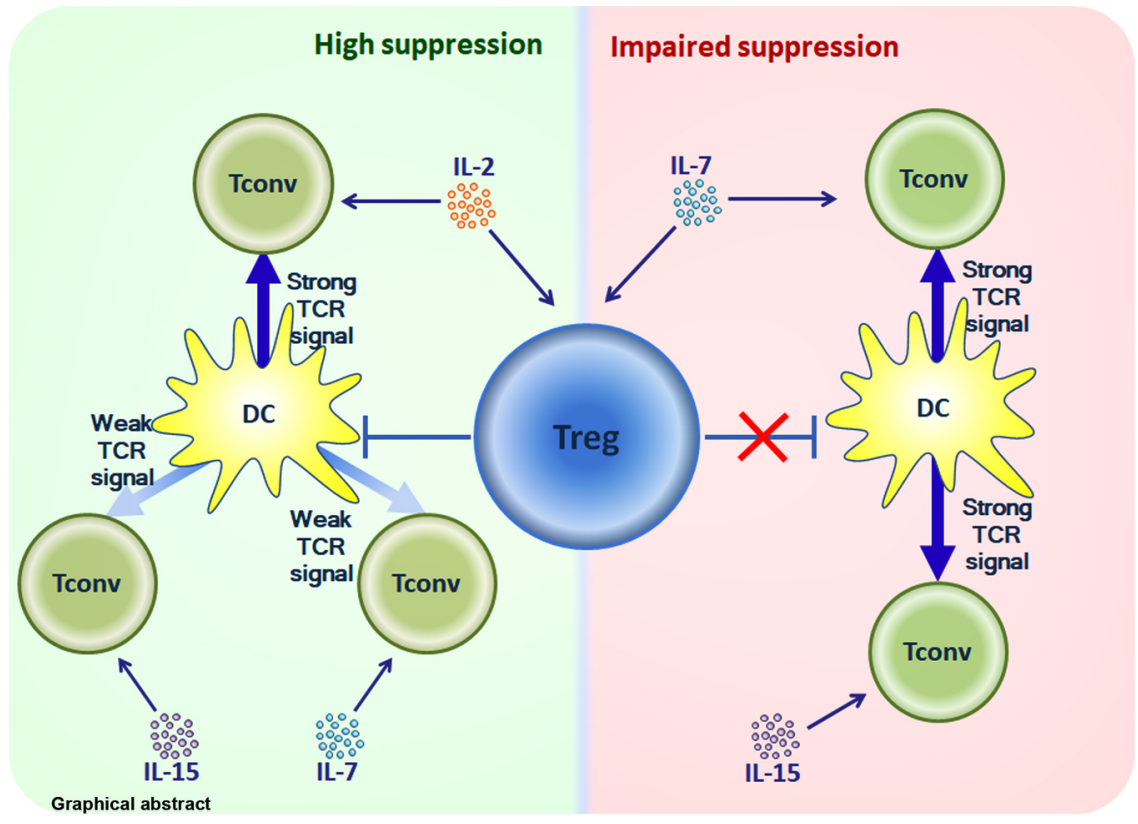

\section{Experiment design}
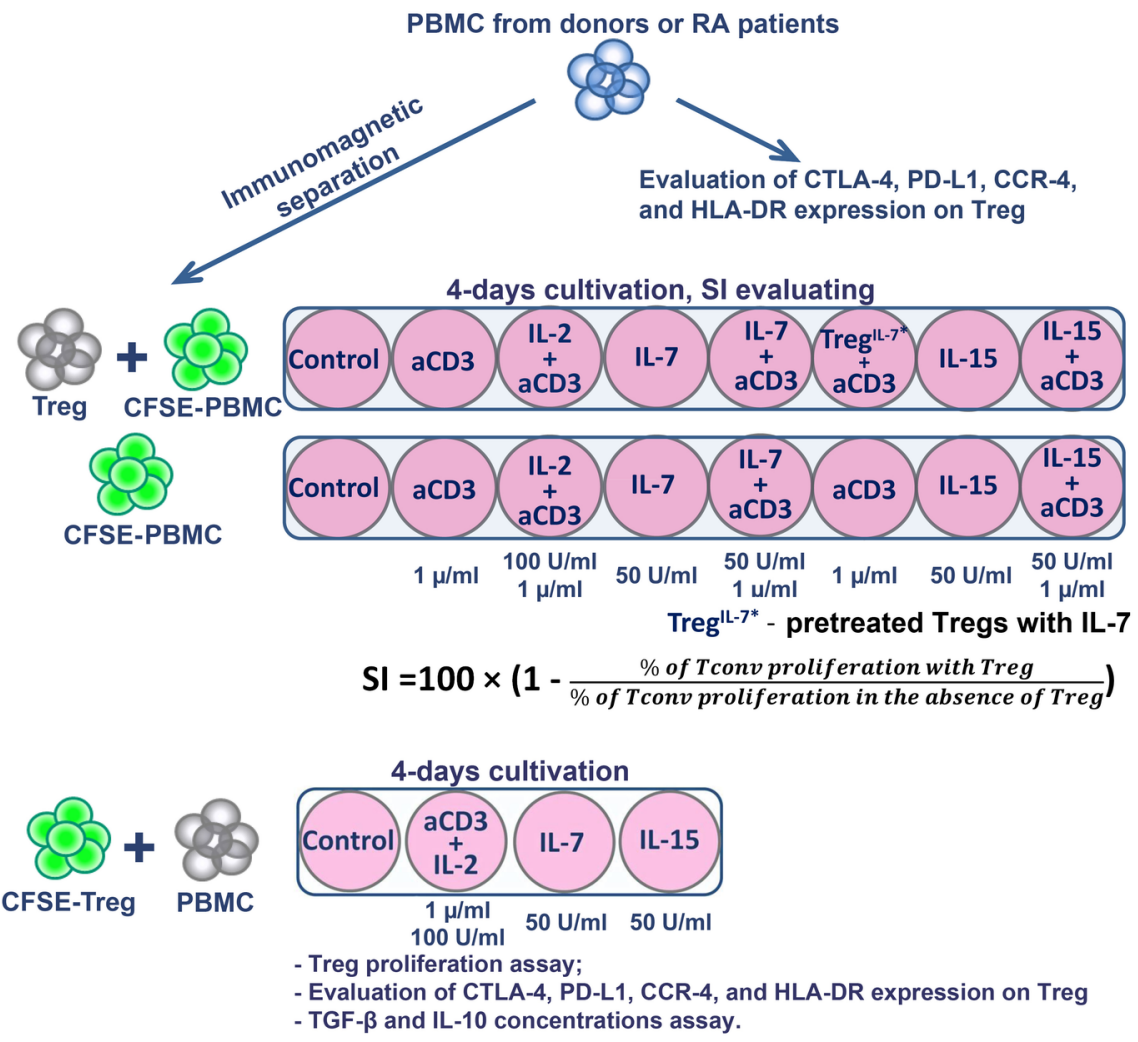

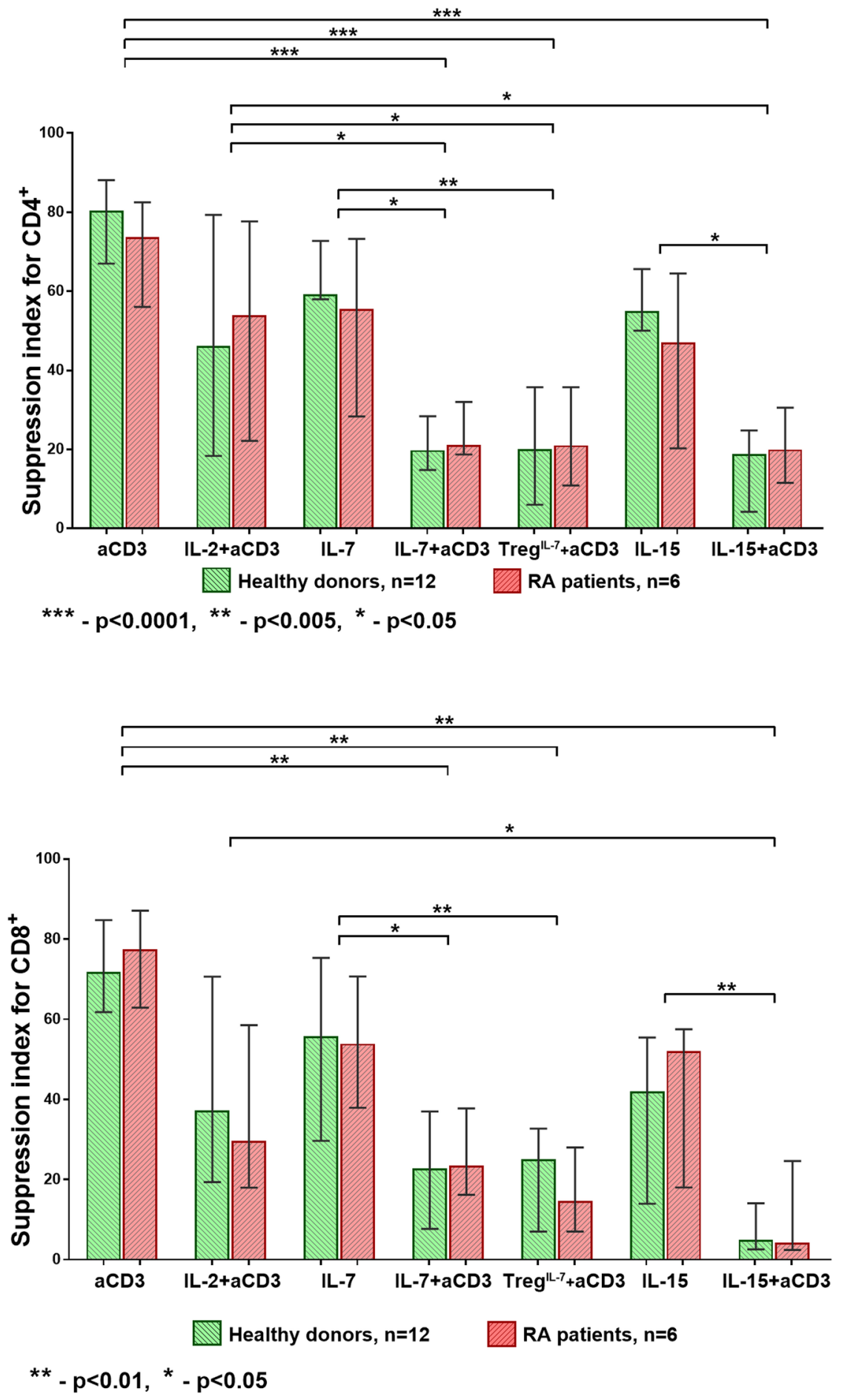

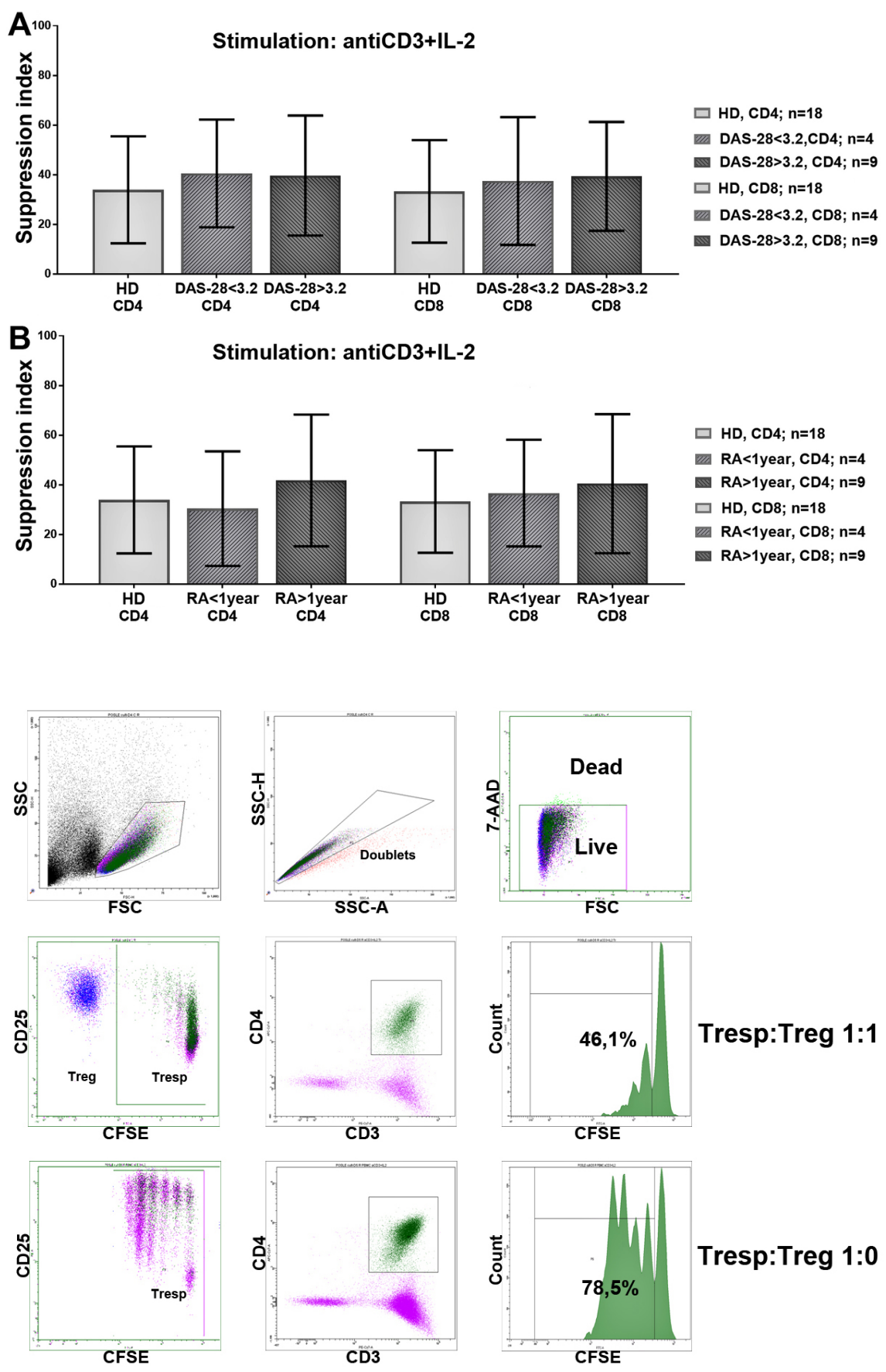

Suppression index, $\mathrm{SI}=100 \times\left(1-\frac{46,1}{78,5}\right)=41,2$ 

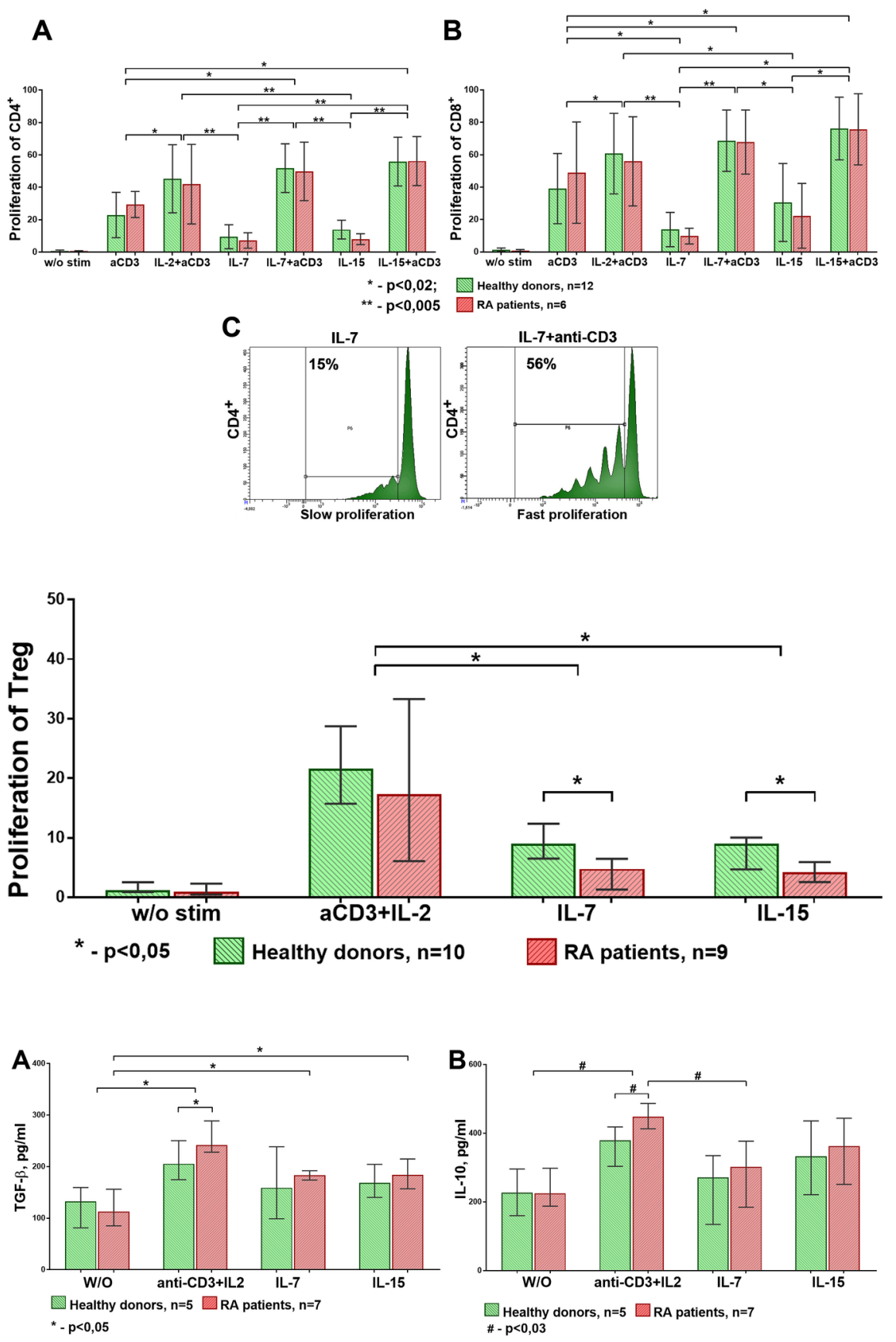

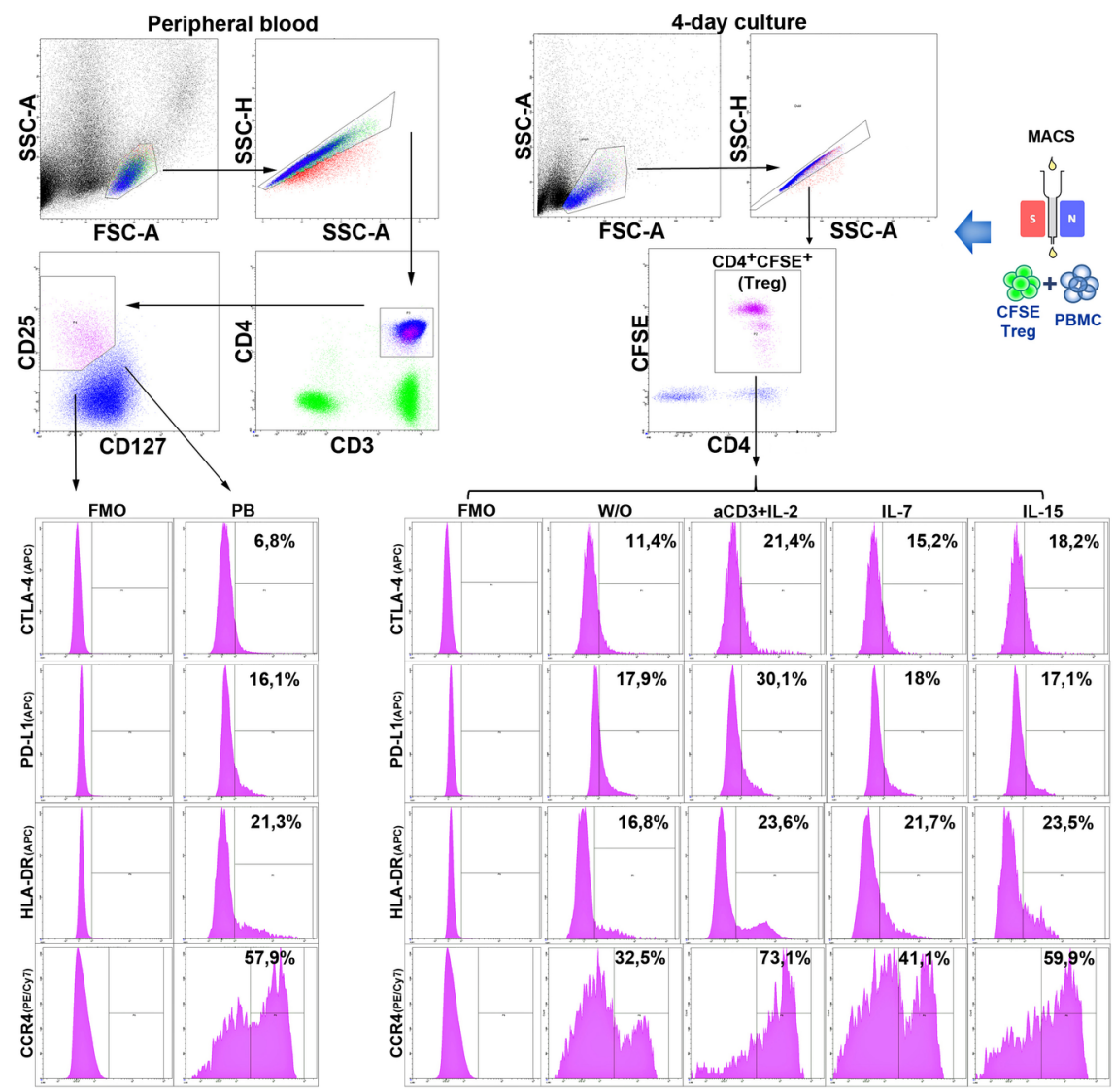

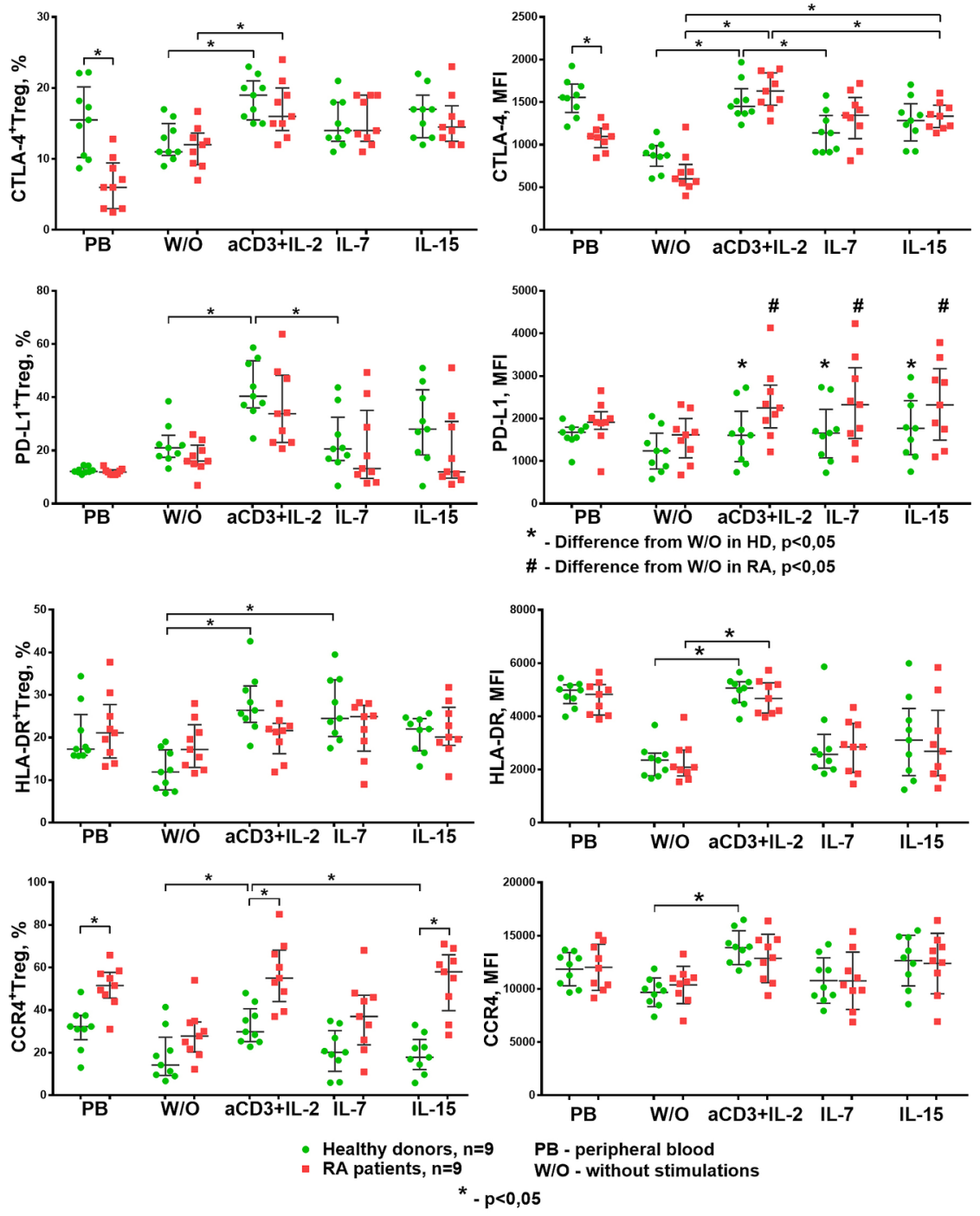